\title{
A heat and mass transfer study of coffee bean roasting
}

\author{
Nabil T. Fadai ${ }^{a}$, John Melrose ${ }^{b, c}$, Colin P. Please $^{a}$, Alexandra Schulman ${ }^{b}$, Robert A. Van Gorder ${ }^{a *}$ \\ ${ }^{a}$ Mathematical Institute, University of Oxford, Andrew Wiles Building, Radcliffe Observatory Quarter, Woodstock Road, Oxford OX2 6GG United Kingdom \\ ${ }^{b}$ Jacobs Douwe Egberts RED UK Ltd, Ruscote Avenue, Banbury OX16 2QU United Kingdom \\ ${ }^{c}$ Koninklijke Douwe Egberts B.V., Oosterdoksstraat 80, 1011 DK Amsterdam Netherlands \\ *Robert.VanGorder@maths.ox.ac.uk
}

\begin{abstract}
Understanding heat, moisture and mass transport during the roasting of a coffee bean is essential to identifying how the colour and flavours are produced. This paper first considers a slightly simplified version of an existing heat and moisture transport model proposed by Fabbri et al. [Numerical modeling of heat and mass transfer during coffee roasting process. Journal of Food Engineering 105 (2011) 264-269], and we show that this model can be fitted well to data for the moisture content of a coffee bean but has some stability issues and lacks some important physical mechanisms. Building on these ideas, a new model is derived from conservation equations. This model is then simplified; in particular, issues of $\mathrm{CO}_{2}$ production are neglected as there is currently insufficient experimental data to fit parameters. This new model is fitted to the same experimental data as presented by Fabbri et al. The new model predicts significantly different internal structure and behaviour of the moisture than the existing model, while both show qualitatively similar average behaviour. This is due to the fact that our model tracks local, rather than bulk, quantities. One benefit to this new model is that it accurately predicts the existence of a sharp drying front, which partitions the bean into an outer dry region and an inner moist region. A detailed comparison of the two models is provided, in order to cast light on the relative importance of various heat and mass transfer mechanisms inherent in coffee bean roasting.
\end{abstract}

Keywords: coffee, roasting, multiphase flow, first principles model, drying front

\section{Introduction}

The coffee industry is worth more than $\$ 100$ billion worldwide per year, making coffee one of the most valuable commodities in the world [20]. One rather fundamental step in coffee production is the roasting of coffee beans. Most of the publications concerning the roasting of coffee beans present experimental data (see e.g. $[1,18,21]$ ), and use regression analysis and simple empirical models to interpret the results. In this paper, we discuss key mechanisms that occur during roasting. These ideas are used to derive a mathematical model which gives a framework that accounts for these and allows solutions to be found that give understanding of the process. In particular, multiphase flow in porous media and mass transfer due to evaporation are seen as important.

Before deriving a type of "first principles" model from conservation equations, we examine the current understanding of roasting of coffee beans in the literature. One of the most detailed models has been developed by [6]. This model consists of a system of partial differential equations (PDEs) describing the transport of moisture and heat throughout a coffee bean. This model, however, has a number of simplifications including the lack of any explicit phase change between liquid water and water vapour in the bean and the assumption that evaporation occurs only at the surface of the coffee bean. Furthermore, the model in [6] uses the concept of "mass diffusivity" to describe the transport of moisture in the coffee bean that was originally derived in [8]. While this may seem appropri- ate, as both papers deal with evaporation in coffee beans, the work in [8] only addresses what happens when coffee beans are dried at temperatures below the boiling temperature of water. When the temperature of water reaches and exceeds its boiling temperature, as it does in the roasting process, other mechanisms can become dominant. In consequence, papers concerning lower-temperature evaporation (such as wood or food drying models, e.g. [17]) are not immediately applicable for higher-temperature evaporation, such as in the roasting of coffee beans.

In this paper, we are motivated to extend and develop the ideas in [6] to derive a mathematical model from first principles using conservation equations. While other aspects of coffee processing have been examined from a mathematical perspective (e.g. [13]), mathematical models describing the roasting of coffee beans has been largely unexplored. The model presented here incorporates the production of carbon dioxide gas, latent heat due to evaporation within the bean, and the changing porosity of the bean. Once a full model is derived, some simplifications are made (in particular, neglecting carbon dioxide production) in order to allow some preliminary understanding of the model behaviour. This indicates that a "drying front" propagates through to the centre of the bean, as well as a ridge of high pressure water vapour near the drying front. Furthermore, by fitting those parameters whose values are not available from elsewhere, we are able to fit this model to the experimental data shown in [6]. 


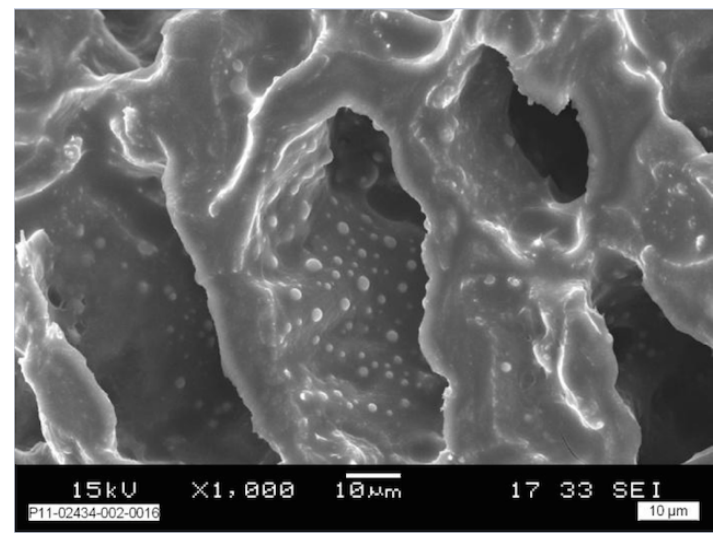

Figure 1: SEM image of the interior of a typical coffee bean after 40 seconds of roasting. Image courtesy of Mondelez International.

\section{Analysis of a Coffee Bean during Roasting}

Before developing a mathematical model of a coffee bean during roasting, it is crucial to understand the physical structure of the coffee bean at a microscopic level and the important mechanisms involved in the roasting process. This section provides a summary of the key points that offer better understanding of the industrial process of roasting coffee beans.

\subsection{The Structure of a Coffee Bean}

A green coffee bean structure can be described as an intercellular matrix consisting of cellulose, glacto-mannans, arabinogalactans, lignin, bound water, and other structural carbohydrates. Within this structure, there are pockets of biological cells containing water, oils, proteins, sugars, cell carbohydrates etc. On roasting, the cell structures are destroyed and many of their contents react through a number of pathways in particular those of the Maillard reactions, to produce a wide variety of reactants, many of which are the flavour and aroma small molecules in addition to some larger polymerised molecules. Structural carbohydrates of the intercellular matrix are also degraded, in part by pyrolysis reactions. $\mathrm{CO}_{2}$ is produced by these reactions and the bean's porosity is increased due to both the destruction of the cells and the degradation of the intercellular matrix.

At the same time, the water within the cells evaporates and becomes water vapour. The resulting high gas pressure causes the cells to expand. This expansion also causes the nano-porous walls of the cells to deform allowing gas to flow through them more easily. Figure 1 shows a typical coffee bean's porous structure after roasting. We can clearly see the pores surrounded by the cellulose wall structure in this scanning electron microscope (SEM) image. Before roasting, these pores contain significant amounts of liquid water, but after roasting the water appears as "spots" on the wall structure of the bean with the rest of the pore filled with gas.

\subsection{The Industrial Roasting Process}

For industrial coffee roasting, two varieties of coffee beans are mainly used: C. arabica and C. robusta. Due to cost and aroma balance, a combination of these varieties is used for the majority of coffee blends. Prior to roasting, both kinds of beans are cleaned and any residual debris removed. Since $C$. robusta beans present different aromas than the $C$. arabica variety, they are sometimes steamed before they are roasted to bring out favourable aromas similar to the $C$. arabica bean.

After any pre-treatment, the beans are roasted normally at around $200^{\circ} \mathrm{C}$. The most common methods used are drum roasters and the fluidised bed roasters [6]. In a drum roaster, the coffee beans are placed in a rotating cylinder, which is attached to a hot air inlet. In this roaster, heat is mainly transferred by conduction from bean-bean contact or from inlet-bean interactions. With the fluidised bed roaster, the beans are placed in a chamber with hot air blown vertically through the bed of beans. This causes the bean to become suspended in the air, and the main heat transfer mechanism in this roaster is convection.

The roasting process can be divided into three phases: drying, flavour and colour development, and cooling. In the drying phase, the evaporation of water from the bean's biological cells brings the moisture content down from an initial value of $12 \%$ to approximately $2 \%$. The bean's temperature rises rapidly, and swelling occurs. This is generally the longest phase of the roast.

Following the drying phase, flavour and colour start to develop in the bean. Once the bean's temperature reaches nearly $200^{\circ} \mathrm{C}$, exothermic reactions in the bean, such as Maillard reactions, can begin. These reactions generate the colour, flavour, and aromas that are typical of roast coffee beans. Additionally, carbon dioxide is generated within the bean, causing the bean to further swell. At some critical point in this stage, one can observe "First Crack". While a detailed physical explanation for First Crack has not yet been found a distinct popping sound can be heard, not unlike the sound of popping popcorn. After First Crack, the bean's colour continues to darken and its aromas continue to enhance. A "Second Crack" can then occur, similar to a snapping sound, when the bean becomes very dark in colour. For industrially-roasted coffee, the roasting process generally ends between First and Second Crack. Before First Crack, the aromas and flavours of the bean have not fully developed, and after Second Crack, the bean is considered to be burnt with all desirable aromas and flavours lost and undesirable aromas generated.

When the required period of roasting has occurred, further roasting is prevented by transferring beans to a second chamber for the cooling phase. Usually, liquid water is added to the chamber (called "quenching") to quickly stop the roasting process. However, some roasters omit the quenching phase and cool the beans using circulated cool air instead. While quenching does add some extra moisture in the bean, a target moisture of less than $5 \%$ is typically achieved. This cooling phase occurs soon after First Crack to achieve a lighter roast, or near Second Crack for a darker roast.

Figure 2 shows how the bean's porous structure changes during roasting. The amount of material decreases during roasting due to evaporation and reactions. We also see that in the bean prior to roasting (a "green" bean), the pores are small while a roasted bean's pores are larger and mostly filled with gas. 


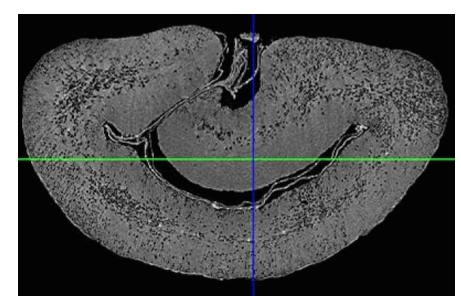

(a)

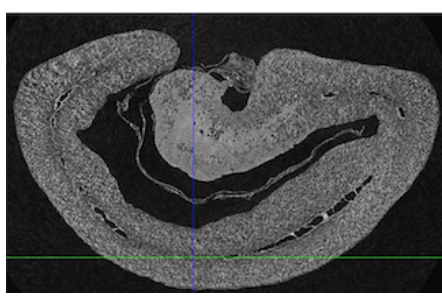

(b)
Figure 2: SEM images of a cross-section of a typical coffee bean before (a) and after (b) roasting. Image courtesy of Mondelez International.

\section{Model 1: Bulk Moisture Content}

We first review a model specifically describing coffee roasting, presented in [6]. It focuses on the heat transport within the bean, and the bulk "moisture" content present within the bean. The moisture is described as one phase with no distinction between vapour and liquid within the bean. The model consists of a coupled system of PDEs with flux boundary conditions which model the transfer of heat and moisture between the bean and the ambient space. The model does not incorporate latent heat within the bean itself but does account for it at the surface. A main consideration of the model is the external geometry of the bean and the PDEs are solved in a realistic shapes by using the commercial package COMSOL [4].

The model presented here is very similar to the model presented in [6], but with a few simplifications. Firstly, all the parameters in the model, except the mass diffusivity $D$, are held constant. Secondly, spherical geometry is used instead of a semi-elliptical geometry or a digitized geometry of a realistic coffee bean. Finally, we will solve the system in MATLAB [12] using a finite difference scheme in space and a stiff ODE solver rather than in COMSOL.

\subsection{Dimensional form of Model 1}

Consider the coupled system of PDEs

$$
\rho_{b} C_{p b} \frac{\partial T^{*}}{\partial t^{*}}+\nabla \cdot\left(-K_{b} \nabla T^{*}\right)=0 \text { and } \frac{\partial c^{*}}{\partial t^{*}}-\nabla \cdot\left(D^{*} \nabla c^{*}\right)=0 .
$$

Here, $T^{*}$ and $c^{*}$ represent the dimensional temperature and moisture content in the bean respectively, and are based on conservation of heat and water. Additionally, the thermal parameters $\rho_{b}, C_{p b}$, and $K_{b}$ refer to the density, specific heat capacity, and thermal conductivity, respectively, of a "bulk" coffee bean. These thermal properties incorporate the fact that a coffee bean contains solid, liquid, and gas phases, and are therefore different than a dry bean. Here we will assume that all thermal parameters of the bean are constants, except for the mass diffusivity term $D^{*}$, which is a strong function of temperature and moisture. In particular, we define

$$
D^{*}\left(T^{*}, c^{*}\right)=\eta_{1} \exp \left(-\frac{E_{a}}{R T^{*}}+\eta_{2} \frac{c_{a v g}^{*}}{c_{0}}\right),
$$

where the term $c_{\text {avg }}^{*}$ denotes the average moisture concentration over the volume $V$ of the bean, i.e. $c_{a v g}^{*}:=\frac{1}{V} \int_{V} c^{*} d V$. This mass diffusivity function is taken directly from [6], with the parameters $\eta_{1}$ and $\eta_{2}$ determined experimentally by [8]. On the surface of the bean, we impose the boundary conditions used by [6]:

$$
\begin{gathered}
K_{b} \nabla T^{*} \cdot \mathbf{n}=h_{m}\left(T_{\infty}-T^{*}\right)+\lambda_{m} D_{m} m_{v} \nabla c^{*} \cdot \mathbf{n}, \\
D^{*} \nabla c^{*} \cdot \mathbf{n}=\beta_{m}\left(c_{\infty}-c^{*}\right),
\end{gathered}
$$

where $\mathbf{n}$ denotes the outward normal to the surface of the bean. Equation (3) means that the thermal flux at the bean's surface is proportional to the difference between the roasting and actual surface temperature, plus the amount of energy needed to evaporate the moisture on surface out of the bean. Equation (4) indicates that the flux of moisture is proportional to the difference between the ambient and the moisture levels on the surface of the bean. The surface moisture diffusivity $D_{m}$ is assumed to be constant and independent of $D^{*}$. Additionally, the convective heat transfer coefficient $h_{m}$, the ambient moisture content and temperature $c_{\infty}, T_{\infty}$, the mass transfer coefficient $\beta_{m}$, and the latent heat of vapourisation $\lambda_{m}$ are all assumed to be constant. Finally, we impose uniform initial conditions for the system, namely,

$$
T^{*}(0, r)=T_{0} \text { and } c^{*}(0, r)=c_{0} \text { for } 0 \leq r^{*} \leq L .
$$

To allow generic behaviour of the model to be determined we assume spherical geometry for the bean with spherical symmetry and the outer surface at $r^{*}=L$. Hence, the solution depends only on $t^{*}$ and $r^{*}$ and is solved in the region $0 \leq r^{*} \leq L$. Additionally, we impose boundedness on $T^{*}$ and $c^{*}$ at the centre of the bean where $r^{*}=0$. This assumption about the geometry will permit us to gain an understanding of the salient features of the model. Model 1 is therefore defined by the PDE system (1), the constitutive equation (2), the boundary conditions (3) and (4), and the initial conditions (5).

\subsection{Non-dimensionalisation of Model 1}

We non-dimensionalise the PDE (1) using the scalings $T^{*}=$ $T_{0}+\left(T_{\infty}-T_{0}\right) T, c^{*}=c_{\infty}+\left(c_{0}-c_{\infty}\right) c, r^{*}=L r, t^{*}=\frac{L^{2} \rho_{b} C_{p b}}{K_{b}} t$, and $D^{*}=D_{0} D$. Here, $D_{0}$ denotes a typical value of the diffusivity; in this case, it was picked as the average diffusivity at the initial and final temperatures with its initial moisture content:

$$
D_{0}=\frac{D^{*}\left(T_{\infty}, c_{0}\right)+D^{*}\left(T_{0}, c_{0}\right)}{2} .
$$

The timescale is the heat diffusion timescale, also known as the Fourier number, which is typically about 130 s. We obtain the non-dimensional system

$$
\frac{\partial T}{\partial t}=\frac{1}{r^{2}} \frac{\partial}{\partial r}\left(r^{2} \frac{\partial T}{\partial r}\right) \text { and } \frac{\partial c}{\partial t}=\frac{\mathscr{D}}{r^{2}} \frac{\partial}{\partial r}\left(r^{2} D \frac{\partial c}{\partial r}\right) .
$$

Here the dimensionless parameter $\mathscr{D}$ denotes the ratio of mass diffusivity to thermal diffusivity, namely, $\mathscr{D}=\frac{D_{0} \rho_{b} C_{p b}}{K_{b}}$, while the non-dimensional mass diffusivity is defined as

$$
\begin{aligned}
D(T, c)=\frac{\eta_{1}}{D_{0}} \exp \left\{-\frac{E_{a} /\left(R T_{0}\right)}{1+\left(\frac{T_{\infty}}{T_{0}}-1\right) T}+\eta_{2} \frac{c_{\infty}}{c_{0}}\right. \\
\left.+3 \eta_{2}\left(1-\frac{c_{\infty}}{c_{0}}\right) \int_{0}^{1} c r^{2} d r\right\} .
\end{aligned}
$$


On the surface of the bean, the boundary conditions in nondimensional form become

$$
\frac{\partial T}{\partial r}=\mathrm{Nu}(1-T)+\xi \frac{\partial c}{\partial r} \text { and } \frac{\partial c}{\partial r}=-\mathrm{Sh} \frac{c}{D} \text { at } r=1
$$

The Nusselt and Sherwood numbers, which are sometimes referred to as the heat and mass transfer Biot numbers, are defined as $\mathrm{Nu}=\frac{h_{m} L}{K_{b}}$ and $\mathrm{Sh}=\frac{\beta_{m} L}{D_{0}}$, respectively, and our final dimensionless group is defined as

$$
\xi=D_{m} \frac{\lambda_{m}\left(c_{0}-c_{\infty}\right) m_{v}}{K_{b}\left(T_{\infty}-T_{0}\right)}
$$

where $m_{v}$ is the molar mass of water. One could interpret $\xi$ as a ratio of surface moisture diffusivity to thermal diffusivity from evaporation. Additionally, we impose Neumann boundary conditions at the centre of the bean, i.e.

$$
\frac{\partial T}{\partial r}=\frac{\partial c}{\partial r}=0 \text { at } r=0 .
$$

Finally, the initial conditions become $T(0, r)=0$ and $c(0, r)=1$ for $0 \leq r \leq 1$.

\subsection{Results}

We solve the non-dimensionalised model presented in Section 3.2 in MATLAB [12] using a central finite volume scheme in the radial component, e.g.

$$
\begin{aligned}
\left.\nabla^{2} T\right|_{r_{i}} \approx \frac{1}{(2 \Delta r)^{2}} & {\left[\left(2+\frac{\Delta r}{r_{i}}\right)^{2}\left(T\left(r_{i+1}, t\right)-T\left(r_{i}, t\right)\right)\right.} \\
& \left.-\left(2-\frac{\Delta r}{r_{i}}\right)^{2}\left(T\left(r_{i}, t\right)-T\left(r_{i-1}, t\right)\right)\right],
\end{aligned}
$$

and a stiff ODE solver for the time component. This finite volume scheme is second-order accurate in theory; in practise, we see a convergence rate of approximately 1.95 at coarser meshes. We use a stiff adaptive ODE solver in time, namely the MATLAB function ode15s, to guarantee that the scheme always stayed in the radius of convergence, as well as 100 spatial meshpoints for good numerical resolution. While other solvers such as COMSOL could have also been used, the transparency of the MATLAB structure and being able to avoid "black-box" solvers made the MATLAB approach desirable to the authors. We use parameter values shown in Table 1 to obtain the results shown in Figures 3 and 4. These parameter values are chosen so that solutions of Model 1 agree with the predictions presented in [6].

As we can see in Figure 4, the predictions shown in [6] can be reproduced well using Model 1. Additionally, we observe from Figure 3 that the main spatial dependence in temperature and moisture content only occur during the first minute of roasting. After the first minute, the bean's temperature and moisture content become nearly spatially uniform.

It is important to note at this point that the boundary condition in $T$ for this model can drive the system unstable. This is due to the fact that we have opposing signs in the boundary condition. For small $t, T \sim 0$ and $c \sim 1$, making $\frac{\partial T}{\partial r} \sim \mathrm{Nu}-1.5 \xi \mathrm{Sh}$.

\begin{tabular}{ccc} 
& & \\
\hline Parameter & Typical Value & Reference(s) \\
\hline$T_{0}$ & $200^{\circ} \mathrm{C}(293.15 \mathrm{~K})$ & Fabbri et al. (2011) \\
$c_{0}$ & $5500 \mathrm{~mol} / \mathrm{m}^{3}($ approx $12 \%$ moisture) & Fabbri et al. (2011) \\
$c_{\infty}$ & $0.5 \mathrm{~mol} / \mathrm{m}^{3}$ & Fabbri et al. (2011) \\
$T_{\infty}$ & $200^{\circ} \mathrm{C}(473.15 \mathrm{~K})$ & Fabbri et al. (2011) \\
$D_{0}$ & $1.63 \times 10^{-10} \mathrm{~m} / \mathrm{m}^{2} / \mathrm{s}$ & Determined from other parameters (Section 3.2) \\
$E_{a}$ & $6.69 \times 10^{4} \mathrm{~J} / \mathrm{mol}$ & Fabbri et al. (2011) \\
$R$ & $8.314 \mathrm{~J} /(\mathrm{mol} \cdot \mathrm{K})$ & Universal gas constant \\
$K_{b}$ & $0.08 \mathrm{~J} /(\mathrm{s} \cdot \mathrm{m} \cdot \mathrm{K})$ & Chosen to agree with simulated data presented in [6] \\
$\rho_{b}$ & $850 \mathrm{~kg} / \mathrm{m}^{3}$ & Chandrasekar and Viswanathan (1999) \\
$C_{p b}$ & $780 \mathrm{~J} /(\mathrm{K} \cdot \mathrm{kg})$ & Chosen to agree with experimental data presented in [6] \\
$L$ & $4 \times 10^{-3} \mathrm{~m}$ & Jacobs Douwe Egberts \\
$\eta_{1}$ & $15.01 \mathrm{~m}^{2} / \mathrm{s}$ & Hernandez-Diaz et al. (2008) \\
$\eta_{2}$ & 1.74 & Hernandez-Diaz et al. (2008) \\
$\lambda_{m}$ & $2.3 \times 10^{6} \mathrm{~J} / \mathrm{kg}$ & Fabbri et al. (2011) \\
$m_{v}$ & $0.018 \mathrm{~kg} / \mathrm{mol}$ & Molar mass of water \\
$D_{m}$ & $5 \times 10^{-10} \mathrm{~m}^{2} / \mathrm{s}$ & Fabbri et al. (2011) \\
$h_{m}$ & $20 \mathrm{~J} /\left(\mathrm{s} \cdot \mathrm{m}^{2} \cdot \mathrm{K}\right)$ & Chosen to agree with experimental data presented in [6] \\
$\beta_{m}$ & $3.4 \times 10^{-6} \mathrm{~m} / \mathrm{s}$ & Chosen to agree with experimental data presented in [6] \\
$\xi$ & $7.9 \times 10^{-3}$ & Determined from other parameters (Section 3.2) \\
$\mathrm{Nu}$ & 1 & Determined from other parameters (Section 3.2) \\
Sh & 1 & Determined from other parameters (Section 3.2) \\
$\mathscr{D}$ & $1.4 \times 10^{-3}$ & Determined from other parameters (Section 3.2) \\
\hline & &
\end{tabular}

Table 1: List of dimensional and non-dimensional parameter values used in Model 1.

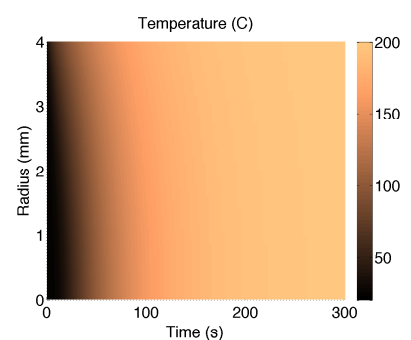

(a)

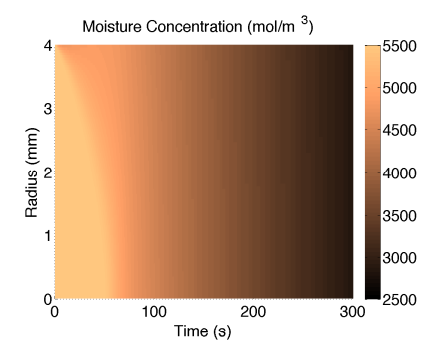

(b)
Figure 3: Plots of a 4mm spherical bean's (a) temperature and (b) moisture concentration for 300 s of roasting at $200^{\circ} \mathrm{C}$ from Model 1 . Parameter values are listed in Table 1.

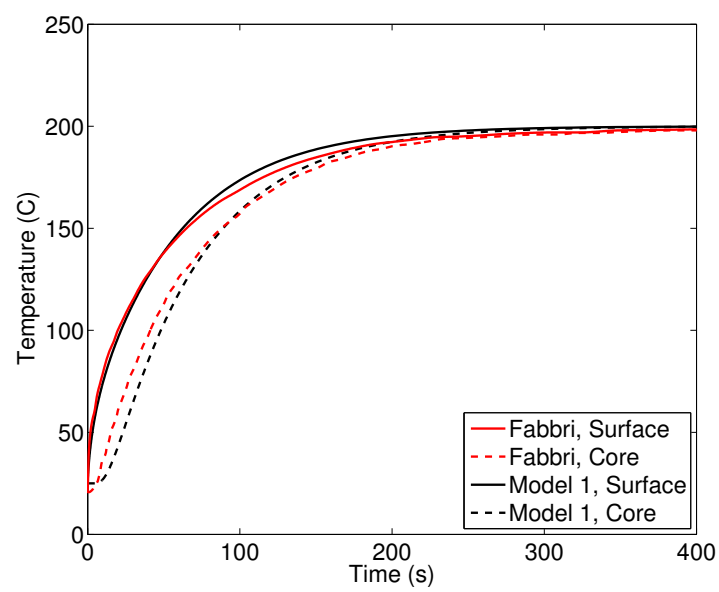

Figure 4: Comparison of temperature curves for a $4 \mathrm{~mm}$ bean at the surface and the centre of the bean during 400 s of roasting at $200^{\circ} \mathrm{C}$. Predictions reproduced from [6] and Model 1. Parameter values for Model 1 are listed in Table 1. 


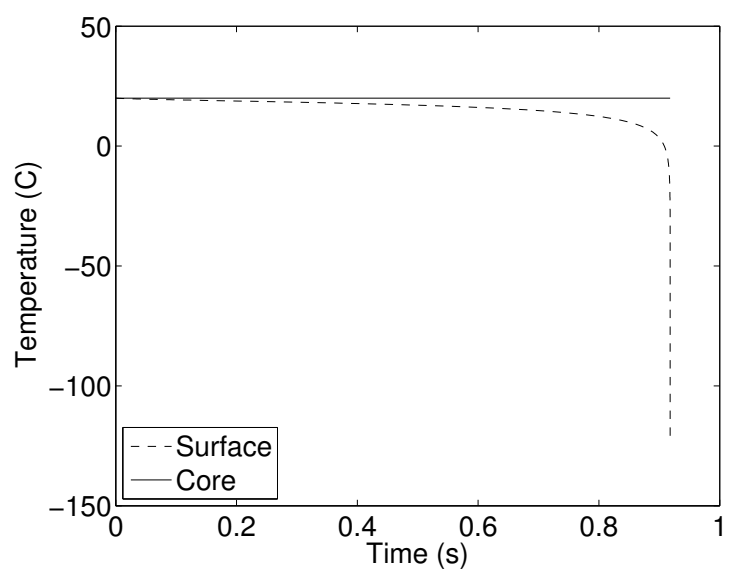

Figure 5: Numerical difficulties are seen when solving Model 1 with $\beta_{m}=$ $3.5 \times 10^{-6} \mathrm{~m} / \mathrm{s}$, giving $\mathrm{Sh}=86$. All other parameters are defined in Table 1 .

Therefore, if $\frac{\partial T}{\partial r}<0$ for long enough time, the surface of the bean will cool the interior rather than heat it. Indeed, if we increase $\beta_{m}$ from $3.4 \times 10^{-6}$ to $3.5 \times 10^{-6}$, we see from Figure 5 that $T$ becomes singular at $t \sim 1 \mathrm{~s}$ when MATLAB tries to solve the system. This may indicate that the boundary value problem consisting of (7) and (9) becomes ill-posed for certain parameter values. Even if this is not the case, Model 1 does appear to give us undue numerical difficulties in some parameter regimes. Further, since certain features of the model are prescribed rather than derived from first principles, troubleshooting these features is difficult. This motivates us to consider a new model derived directly from conservation equations, which we do in the next section.

\section{Model 2: Local Moisture Content}

Model 1 is a good model of the observed behaviour during coffee roasting, as parameters and model elements were specifically chosen for the roasting process. However, the fact that the moisture content was treated as a bulk quantity without specifying the phase state at any point in the bean makes the model limited. This also meant that the bulk thermal properties of the bean may be oversimplified for the roasting process. Additionally, latent heat and evaporation effects can happen in the interior of the bean, since the centre of the bean goes above the boiling temperature of water, and therefore such effects need to be incorporated in the model.

Using the bulk roasting model of [6] as motivation, in this section we derive a roasting model from first principles. Such a model has not previously been considered for coffee bean roasting, although we should remark that similar mathematical modelling that incorporates multiphase flow has been considered for other similar applications, including baking of bread [23], plasma/soil flow and heat transfer in an electric arc furnace [15], burning of timber [16], fire propagation in heterogeneous combustible media [11], and modelling forest fires [19]. Such a model should incorporate multi-phase flow and $\mathrm{CO}_{2}$ production. We assume that no deformation of the bean occurs during the roasting process. While deformation of the bean plays a

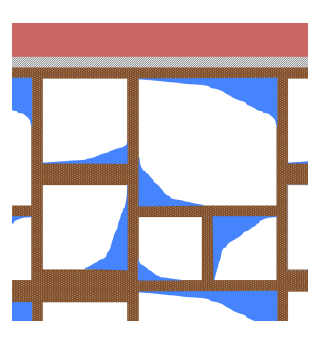

(a)

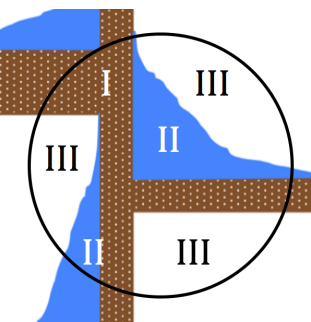

(b)

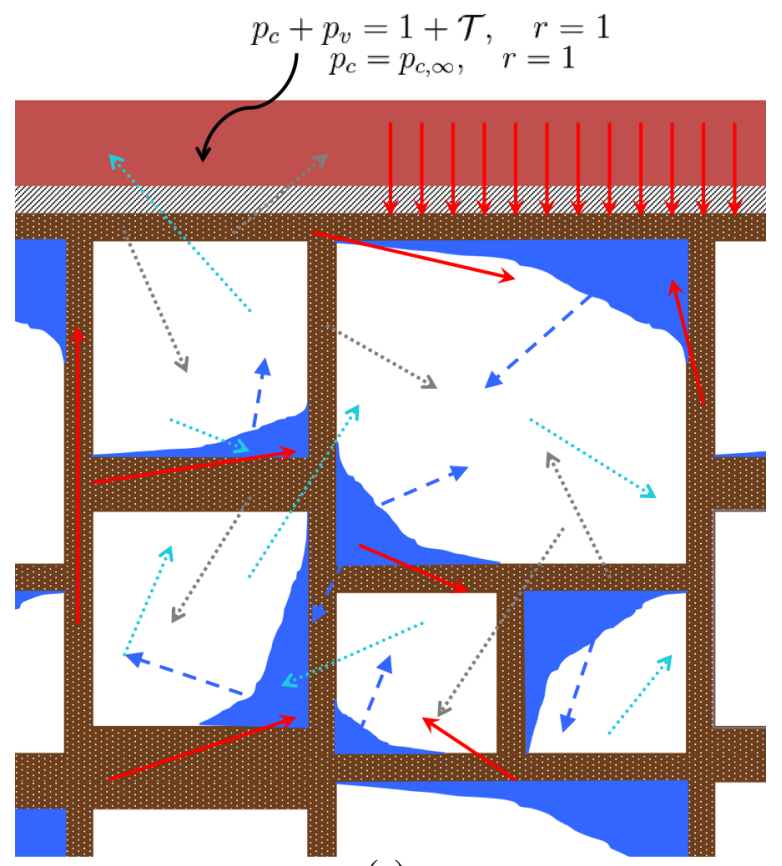

(c)

Figure 6: Schematic representation of a coffee bean structure. (a) The exterior of the bean is shown in red, the surface of the bean is represented by the striped region, and the biological cells are shown as white squares partially filled with blue water. (b) The representative volume of interest is marked by a black circle, with each phase (I, II, and III) labeled. (c) The complete diagram for Model 2 with boundary conditions. The dark blue arrows represent liquid water transport, the light blue arrows represent water vapour transport, the grey arrows represent $\mathrm{CO}_{2}$ transport, and the red arrows represent thermal activity.

significant role in the roasting of coffee beans, it was omitted in this first attempt at a model. We also assume that the density of the solid coffee bean and the liquid water are constant.

To aid the derivation process, a simplified diagram (Figure 6(a)) shows the different phases existing within a coffee bean. The white squares represent the pockets containing the biological cells, which are partially filled with liquid water (shown in blue). The solid nano-porous structure is shown in brown, and the surface of the bean is represented by the striped region near the top of the figure. Finally, the exterior of the bean, i.e. the roasting chamber, is represented by the red region at the top of the figure.

\subsection{Derivation of Dimensional Model 2}

\subsubsection{Introduction of Multiphase Components}

Consider a representative small volume of interest with three phases present: solid (I), liquid (II), and gas (III). In the solid 
phase, only the coffee bean structure consisting of cellulose and other organic molecules exists. In the liquid phase, only liquid water is present. Finally, in the gas phase, water vapour and $\mathrm{CO}_{2}$ coexist. Additionally, we will assume that the temperature in all phases is identical for a given volume of interest (i.e. $T_{i}^{*}=$ $T^{*}$, for each phase $i$ ). These phases can be summarized using Figure 6(b), where the volume of interest is shown within a black circle, with each phase marked.

We define the porosity, $\phi$, as the ratio of the total volume the gas and liquid phases occupy, to the total representative volume. Similarly, we define the saturation, $S$, as the the volume fraction of liquid water divided by the total volume of liquid water and gas. Therefore, we have

$$
\phi=\frac{V_{\mathrm{II}+\mathrm{III}}}{V_{\mathrm{I}+\mathrm{II}+\mathrm{III}}} \text { and } S=\frac{V_{\mathrm{II}}}{V_{\mathrm{II}+\mathrm{III}}}=\frac{V_{\mathrm{II}}}{\phi V_{\mathrm{I}+\mathrm{II}+\mathrm{III}}} .
$$

Using these two non-dimensional quantities, we can define the volume fractions of phases I, II, and III as $1-\phi, \phi S$, and $\phi(1-$ $S$ ), respectively.

\subsubsection{Conservation of Mass (Solid Phase)}

We begin by using conservation of mass for the four species present: solid coffee bean, liquid water, water vapour, and $\mathrm{CO}_{2}$ gas. For the solid phase, there is no movement in the particles (and hence, no flux), but the particles in the coffee bean structure will react to produce $\mathrm{CO}_{2}$ gas. This can be described as

$$
\frac{\partial}{\partial t^{*}}\left((1-\phi) \rho_{s}\right)=-I_{c}^{*}
$$

where $I_{c}^{*}$ is the production rate of $\mathrm{CO}_{2}$ gas. In other words, the porosity of the bean will increase as more $\mathrm{CO}_{2}$ gas is produced. As a visual aid, a schematic representation of this equation is given in Figure 6(c), where the grey arrows represent the production and transport of $\mathrm{CO}_{2}$ gas.

\subsubsection{Conservation of Mass (Liquid Phase)}

For the liquid phase, we must consider the loss of liquid water due to evaporation as well as the mass flux of water, $\mathbf{j}_{w}$, to incorporate diffusion. This can be summarized as

$$
\frac{\partial}{\partial t^{*}}\left(\phi S \rho_{w}\right)+\nabla \cdot \mathbf{j}_{w}=-I_{v}^{*}
$$

where $I_{v}^{*}$ refers to the evaporation rate of liquid water. This means that the local saturation can increase or decrease based on local condensation or evaporation, as well as any movement of liquid from mass flux. Referring to Figure 6(c), the transport of water is represented by the dark blue dashed arrows.

\subsubsection{Conservation of Mass (Gas Phase)}

In the gas phase, we incorporate mass fluxes $\mathbf{j}_{v}$ and $\mathbf{j}_{c}$ to allow for the transport of water vapour and $\mathrm{CO}_{2}$ gas, as well as account for the production rates of $\mathrm{CO}_{2}$ gas and water vapour. Additionally, we use the ideal gas law to define $\rho_{v}=p_{v} m_{v} / R T^{*}$ and $\rho_{c}=p_{c} m_{c} / R T^{*}$, where $m_{v}$ and $m_{c}$ are the molar masses of water and $\mathrm{CO}_{2}$ respectively, and $p_{v}$ and $p_{c}$ are the partial pressures of water vapour and $\mathrm{CO}_{2}$ gas respectively. This gives us

$$
\begin{aligned}
& \frac{\partial}{\partial t^{*}}\left(\phi(1-S) \frac{p_{v}^{*} m_{v}}{R T^{*}}\right)+\nabla \cdot \mathbf{j}_{v}=I_{v}^{*}, \\
& \frac{\partial}{\partial t^{*}}\left(\phi(1-S) \frac{p_{c}^{*} m_{c}}{R T^{*}}\right)+\nabla \cdot \mathbf{j}_{c}=I_{c}^{*} .
\end{aligned}
$$

Similar to the liquid water equation, these mass conservation equations for the gas species tell us that the partial pressure of a gas will change based on any gas production in the solid or liquid phases, as well as any diffusion through the nano-porous structure. Again, we can refer to Figure 6(c) which represents the transport of water vapour (shown in light blue) and $\mathrm{CO}_{2}$ gas (shown in grey).

\subsubsection{Derivation of Mass Flux Terms}

The mass fluxes of gases are due to the total gas pressure gradient and binary diffusion ([2], [14]). Additionally, the liquid water transport is due to the water pressure gradient, which is assumed to be equal to the total gas pressure gradient, and moisture diffusion ([2], [14]). We take these directly from [23] using

$$
\begin{gathered}
\mathbf{j}_{v}^{*}=-\frac{\rho_{v}^{*} k_{g}^{*}}{\mu_{g}^{*}} \nabla\left(p_{v}^{*}+p_{c}^{*}\right)-m_{v} M^{*} D_{\mathrm{eff}, \mathrm{g}}^{*} \nabla \chi_{v}, \\
\mathbf{j}_{c}^{*}=-\frac{\rho_{c}^{*} k_{g}^{*}}{\mu_{g}^{*}} \nabla\left(p_{v}^{*}+p_{c}^{*}\right)-m_{c} M^{*} D_{\mathrm{eff}, \mathrm{g}}^{*} \nabla \chi_{c}, \\
\mathbf{j}_{w}^{*}=-\frac{\rho_{w} k_{w}^{*}}{\mu_{w}^{*}} \nabla\left(p_{v}^{*}+p_{c}^{*}\right)-\rho_{w} D_{w}^{*} \nabla\left(\frac{\phi S \rho_{w}}{(1-\phi) \rho_{s}}\right) .
\end{gathered}
$$

Here, $k_{g}$ and $k_{w}$ denote the permeabilities of gas and water within the nano-porous coffee bean structure, while $\mu_{g}$ and $\mu_{w}$ represent the dynamic viscosities of gas and water in the bean. Additionally, $D_{\text {eff,g }}^{*}$ and $D_{w}^{*}$ are the effective diffusivities of gas and liquid water due to "standard" diffusion, i.e. Fick's Law ([7]).

It is important to note that $M^{*}$ denotes the molar density of the gas mixture, i.e. $M^{*}=\rho_{v}^{*} / m_{v}+\rho_{c}^{*} / m_{c}=\frac{p_{v}^{*}+p_{c}^{*}}{R T^{*}}$. Since the molar fractions $\chi_{v}$ and $\chi_{c}$ sum to one, we can substitute $\nabla \chi_{c}=-\nabla \chi_{v}$ and, from the definition of the molar fraction, we have

$$
\chi_{v}=\frac{\rho_{v}^{*} / m_{v}}{\rho_{v}^{*} / m_{v}+\rho_{c}^{*} / m_{c}}=\frac{p_{v}^{*}}{p_{v}^{*}+p_{c}^{*}} .
$$

More care would need to be taken with molar fraction should a third gas species be included later. The second term in $\mathbf{j}_{w}$ represents an "unforced" diffusion term of liquid water, which is driven by any change in moisture content. Here, moisture content is defined as the mass ratio of liquid water to dry solids. Using our definitions of volume fractions in (13), we can define moisture content as $\phi S \rho_{w} /(1-\phi) \rho_{s}$.

\subsubsection{Conservation of Energy}

We note, from $[9,22,23]$, that each phase $i$ contains enthalpy $\rho_{i}^{*} C_{p i} V_{f, i} T^{*}$ and contributes a diffusive heat flux $-K_{i} V_{f, i} \nabla T^{*}$. Additionally, from [9, 22, 23], advection of the enthalpy in phase $i$ is given by $\mathbf{v}_{i} \rho_{i} C_{p i} V_{f, i} T^{*}$. Here, $V_{f, i}$ represents the volume fraction for each phase and the velocity field $\mathbf{v}_{i}$ is given by 
$\mathbf{v}_{i}=\frac{\mathbf{j}_{i}}{\rho_{i}}$. Finally, we note that the energy contributing to production of $\mathrm{CO}_{2}$ gas and evaporation of liquid water is given by $\lambda_{c} I_{c}^{*}$ and $\lambda_{v} I_{v}^{*}$ respectively, where $\lambda_{v}$ is the latent heat of vapourisation of water and $\lambda_{c}$ denotes the energy (per $\mathrm{kg}$ of solid phase) required for the solid phase to react and produce $\mathrm{CO}_{2}$ gas. Since we do not track the fine scale structure of the bean, it makes sense to consider a locally volume averaged formulation, in the standard way $[9,22]$. This can be summarised by

$$
\begin{aligned}
\frac{\partial}{\partial t^{*}}\left(\sum_{i} \rho_{i}^{*} C_{p i} V_{f, i} T^{*}\right) & +\nabla \cdot\left(\sum_{i} \mathbf{v}_{i} \rho_{i} C_{p i} V_{f, i} T^{*}\right) \\
& -\nabla \cdot\left(\sum_{i} K_{i} V_{f, i} \nabla T^{*}\right)=-\lambda_{v} I_{v}^{*}-\lambda_{c} I_{c}^{*} .
\end{aligned}
$$

All of these thermal processes can be summarised with a schematic representation given in Figure 6(c), where the red arrows denote thermal activity.

\subsubsection{Derivation of Evaporation and Production Rates}

For the evaporation rate $I_{v}^{*}$, we use Langmuir's equation [10] based on the difference between the actual partial pressure and the equilibrium partial pressure, given by

$$
I_{v}^{*}=\left(p_{S T}^{*}\left(T^{*}\right)-p_{v}^{*}\right) \sqrt{\frac{m_{v}}{2 \pi R T^{*}}} \frac{\text { Surface Area }}{\text { Volume }} .
$$

This equilibrium pressure $p_{S T}^{*}\left(T^{*}\right)$ can be calculated using a steam table. However, we need to also incorporate the fact that evaporation occurs at the interface between the liquid and gas phases. The size of this interface is dictated by the surfaceto-volume ratio. For example, if either of their related volume fractions should be zero, evaporation cannot occur. If we assume that the geometry of the liquid phase is roughly spherical, then we can approximate this ratio as

$$
\frac{\text { Surface Area }}{\text { Volume }}=\frac{3}{\delta} V_{f, \mathrm{II}} V_{f, \mathrm{III}}=\frac{3}{\delta} \phi^{2} S(1-S),
$$

where $\delta$ is a typical radius of a biological cell. Combining all of these terms together gives us

$$
I_{v}^{*}=3 \phi^{2} S(1-S) \frac{p_{S T}^{*}\left(T^{*}\right)-p_{v}^{*}}{\delta} \sqrt{\frac{m_{v}}{2 \pi R T^{*}}} .
$$

The steam table pressure for pure water can be approximated by, from [5],

$$
p_{S T}^{*}\left(T^{*}\right)=A_{1} \exp \left(A_{2}-\frac{A_{3}}{T^{*}}\right)
$$

For $I_{c}^{*}$, the kinetics of the $\mathrm{CO}_{2}$-producing reaction are not as obvious as the evaporation rate. If one assumes that this reaction can only take place on the interface between the solid and gas phases, then we could approximate this production rate using Langmuir's equation [10] as

$$
I_{c}^{*}=3 \phi(1-\phi)(1-S) \frac{p_{e q}^{*}\left(T^{*}\right)-p_{c}^{*}}{\delta} \sqrt{\frac{m_{c}}{2 \pi R T^{*}}} .
$$

The equilibrium pressure of this reaction, $p_{e q}^{*}\left(T^{*}\right)$, should only allow the reaction to proceed in a single direction (i.e. no solid can be created from $\mathrm{CO}_{2}$ gas). Additionally, it is quite possible that the reaction can happen internally within the coffee bean, or that the geometry at the interface of these two phases suggests a different surface-to-volume ratio. In either case, or for other factors that have not been incorporated in this model, the expression for $I_{c}^{*}$ would need to be modified.

\subsection{Boundary Conditions for Model 2}

For the heat boundary condition, we assume that the principal means of heat transport away from the boundary is due to convection. This gives rise to our dimensional boundary condition in $T^{*}$ on the surface of the bean, ${ }^{1}$

$$
\left(\sum_{i} K_{i} V_{f, i} \nabla T^{*}\right) \cdot \mathbf{n}=\left(\sum_{i} h_{i} V_{f, i}\right)\left(T_{\infty}-T^{*}\right) .
$$

Additionally, it is quite possible that there are additional heat fluxes to consider, notably a term involving the flux of liquid water. For this model, we will assume that evaporation of liquid water occurs only inside the bean and not directly on the surface. Therefore, a flux term due to moisture, like the one used in Model 1, is not included.

For our boundary condition for the gases, we assume that the number of moles of gas in the roasting chamber $(n)$, as well as the volume of the roasting chamber itself $(V)$, stay constant during the roasting process, i.e. $\frac{\sum_{i} p_{i}^{*}}{T^{*}}=\frac{n R}{V}$. By ideal gas law, this means that the ratio of roasting temperature to initial temperature $\left(\frac{T_{\infty}}{T_{0}}\right)$ is identical to the ratio of the total gas pressure in the roasting chamber to the initial total gas pressure, $\frac{\sum_{i} p_{i}^{*}}{p_{0}}$. Additionally, this means that we must also declare one of the partial pressures of gas at the surface of the bean; for this model, we choose to declare the partial pressure of $\mathrm{CO}_{2}$ gas at the boundary. This is equivalent to saying that

$$
p_{c}^{*}+p_{v}^{*}=\frac{T_{\infty}}{T_{0}} p_{0} \text { and } p_{c}^{*}=p_{c, \infty}^{*} \text { at } r^{*}=L .
$$

Since the beans have been stored at ambient temperature and a constant environment before going in to the roaster, we impose spatially uniform initial conditions for the system, namely, $T^{*}=T_{0}$, atmospheric vapour pressure $\left(p_{v}^{*}=p_{v 0}^{*}\right)$, and $\mathrm{CO}_{2}$ pressure $\left(p_{c}^{*}=p_{c 0}^{*}\right)$.

The complete model is represented in Figure 6(c). Dimensional parameters (and typical values) are given in Table 2.

\subsection{Non-dimensionalisation of Model 2}

We non-dimensionalise the variables in the PDE system using: $T^{*}=T_{0}+\left(T_{\infty}-T_{0}\right) T, p_{v}^{*}=p_{0} p_{v}, p_{c}^{*}=p_{0} p_{c}, r^{*}=L r$,

\footnotetext{
${ }^{1}$ It is important to note that greater care may be needed for this flux condition. For instance, the roasting chamber is assumed to only contain gas, and therefore might not be reasonable to contain term with volume fractions like $h_{i} V_{f, i}$. In consequence, it may be more realistic to have a "bulk" convective coefficient; however, this is omitted from our model.
} 


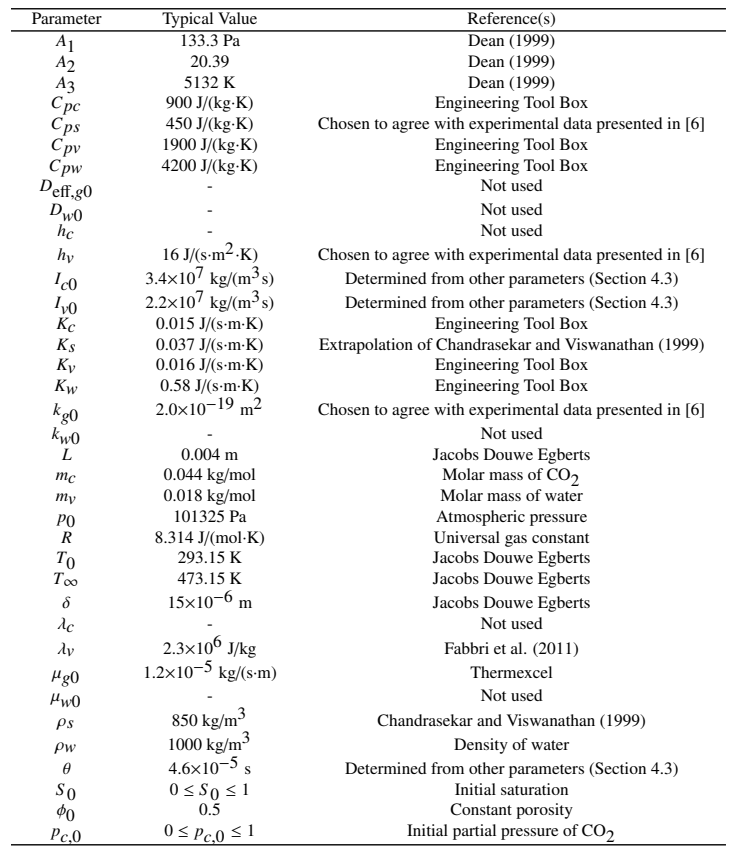

Table 2: Typical values for dimensional parameters used in Model 2. Engineering Tool Box values are from http://www.engineeringtoolbox.com/ (10July-2015). Thermexcel values are from http://www.thermexcel.com/ (10-July2015).

and $t^{*}=\theta t$, and similarly all dimensional functions are nondimensionalised by dividing by a typical function value, i.e. $f^{*}=f_{0} f$. The timescale, $\theta$, is chosen to be the time for evaporation so that $\theta=\frac{\rho_{w}}{I_{v 0}}$, where $I_{v 0}=\frac{3 p_{0}}{\delta} \sqrt{\frac{m_{v}}{2 \pi R T_{0}}}$.

The full model in spherical coordinates can then be written in the following form. First, the mass conservation equations become

$$
\frac{\partial \phi}{\partial t}=\frac{\kappa_{1}}{\alpha_{1}} I_{c}
$$

$$
\frac{\partial}{\partial t}(\phi S)=-I_{v}+\kappa_{3} \mathscr{D}_{3} \nabla \cdot\left(\frac{k_{w}}{\mu_{w}} \nabla\left(p_{v}+p_{c}\right)+\frac{1}{\alpha_{1}} \mathscr{D}_{2} \nabla\left(\frac{\phi S}{1-\phi}\right)\right),
$$

$$
\begin{aligned}
& \frac{\partial}{\partial t}\left(\frac{\phi(1-S) p_{v}}{1+\mathscr{T} T}\right)=\frac{1}{\alpha_{2}} I_{v}+\mathscr{D}_{3} \nabla \cdot\left(\frac{k_{g}}{\mu_{g}} \frac{p_{v}}{1+\mathscr{T} T} \nabla\left(p_{v}+p_{c}\right)\right. \\
&\left.+\mathscr{D}_{1} \frac{\left(p_{v}+p_{c}\right) D_{\mathrm{eff}, \mathrm{g}}}{1+\mathscr{T} T} \nabla\left(\frac{p_{v}}{p_{v}+p_{c}}\right)\right)
\end{aligned}
$$

$$
\begin{gathered}
\frac{\partial}{\partial t}\left(\frac{\phi(1-S) p_{c}}{1+\mathscr{T} T}\right)=\frac{\kappa_{1} \kappa_{2}}{\alpha_{2}} I_{c}+\mathscr{D}_{3} \nabla \cdot\left(\frac{k_{g}}{\mu_{g}} \frac{p_{c}}{1+\mathscr{T} T} \nabla\left(p_{v}+p_{c}\right)\right. \\
\left.-\mathscr{D}_{1} \frac{\left(p_{v}+p_{c}\right) D_{\mathrm{eff}, \mathrm{g}}}{1+\mathscr{T} T} \nabla\left(\frac{p_{v}}{p_{v}+p_{c}}\right)\right) .
\end{gathered}
$$

The energy conservation equation becomes

$$
\begin{aligned}
\frac{\partial}{\partial t}(\text { Enthalpy }) & -\nabla \cdot(\text { Advection })= \\
& -\gamma I_{v}-\gamma \kappa_{1} \kappa_{4} I_{c}+\mathscr{T} \nabla \cdot(\text { Conduction }),
\end{aligned}
$$

\begin{tabular}{ccc}
\hline $\begin{array}{c}\text { Dimensionless } \\
\text { Parameter }\end{array}$ & $\begin{array}{c}\text { Relationship to Dimensional } \\
\text { Parameters }\end{array}$ & Typical value of Parameter \\
\hline$\alpha_{1}$ & $\frac{\rho_{s}}{\rho_{w}}$ & \\
$\alpha_{2}$ & $\frac{p_{0} m_{v}}{\rho_{w} R T_{0}}$ & 0.85 \\
$B_{1}$ & $\frac{A_{1}}{\rho_{0}} \exp \left(A_{2}-\frac{A_{3}}{T_{0}}\right)$ & $0.5 \times 10^{-4}$ \\
$B_{2}$ & $\frac{A_{3}}{T_{0}}$ & 17.5 \\
$\gamma$ & $\frac{\lambda_{v}}{T_{0} C_{p w}}$ & 1.9 \\
$\zeta_{1}$ & $\frac{K_{S}}{\rho_{w} C_{p w}} \cdot \frac{\rho_{w}}{L^{2} I_{v 0}}$ & $2.4 \times 10^{-8}$ \\
$\zeta_{2}$ & $\frac{K_{w}}{\rho_{w} C_{p w}} \cdot \frac{\rho_{w}}{L^{2} \nu_{v 0}}$ & $3.9 \times 10^{-7}$ \\
$\zeta_{3}$ & $\frac{K_{v}}{\rho_{w} C_{p w}} \cdot \frac{\rho_{w}}{L^{2} I_{v 0}}$ & $1.1 \times 10^{-8}$ \\
$\mathscr{C}_{1}$ & $\frac{C C_{p s}}{C_{p w}}$ & 0.11 \\
$\mathscr{C}_{2}$ & $\frac{C_{p v}}{C_{p w}}$ & 0.45 \\
$\mathscr{D}_{3}$ & $\frac{k_{g 0} p_{0}}{\mu_{g 0}} \cdot \frac{\rho_{w}}{L^{2} I_{v 0}}$ & $4.7 \times 10^{-9}$ \\
$\mathscr{T}$ & $\frac{T_{\infty}}{T_{0}}-1$ & 0.61 \\
$\mathrm{Nu}_{v}$ & $\frac{h_{v} L}{K_{v}}$ & 4 \\
\hline
\end{tabular}

Table 3: Description and typical values of dimensionless parameters used in the simplified form of Model 2. Dimensional parameter values are given in Table 2.

where

$$
\begin{aligned}
& \text { Enthalpy }=\alpha_{1} \mathscr{C}_{1}(1-\phi)(1+\mathscr{T} T)+\phi S(1+\mathscr{T} T) \\
& +\alpha_{2} \mathscr{C}_{2} \phi(1-S) p_{v}+\frac{\alpha_{2} \mathscr{C}_{3}}{\kappa_{2}} \phi(1-S) p_{c}
\end{aligned}
$$

$$
\begin{gathered}
\text { Advection }=\kappa_{3} \mathscr{D}_{3} \phi S(1+\mathscr{T} T)\left(\frac{k_{w}}{\mu_{w}} \nabla\left(p_{v}+p_{c}\right)\right. \\
\left.+\frac{1}{\alpha_{1}} \mathscr{D}_{2} \nabla\left(\frac{\phi S}{1-\phi}\right)\right) \\
+\alpha_{2} \mathscr{C}_{2} \mathscr{D}_{3} \phi(1-S)\left(\frac{k_{g}}{\mu_{g}} p_{v} \nabla\left(p_{v}+p_{c}\right)\right. \\
\left.+\mathscr{D}_{1}\left(p_{v}+p_{c}\right) D_{\mathrm{eff}, \mathrm{g}} \nabla\left(\frac{p_{v}}{p_{v}+p_{c}}\right)\right) \\
+\frac{\alpha_{2} \mathscr{C}_{3} \mathscr{D}_{3}}{\kappa_{2}} \phi(1-S)\left(\frac{k_{g}}{\mu_{g}} p_{c} \nabla\left(p_{v}+p_{c}\right)\right. \\
\left.-\mathscr{D}_{1}\left(p_{v}+p_{c}\right) D_{\mathrm{eff}, \mathrm{g}} \nabla\left(\frac{p_{v}}{p_{v}+p_{c}}\right)\right),
\end{gathered}
$$

Conduction $=\left(\zeta_{1}(1-\phi)+\zeta_{2} \phi S+\zeta_{3} \phi(1-S)+\zeta_{4} \phi(1-S)\right) \nabla T$.

The heat flux condition, (28), becomes

$$
\frac{\partial T}{\partial r}=\mathrm{Nu}_{v} \zeta_{3}(1-T) \frac{\phi(1-S)(1+H)}{\zeta_{1}(1-\phi)+\zeta_{2} \phi S+\left(\zeta_{3}+\zeta_{4}\right) \phi(1-S)},
$$

while the concentration boundary conditions are

$$
p_{c}+p_{v}=1+\mathscr{T} \text { and } p_{c}=p_{c, \infty} \text { at } r=1
$$

Additionally, because of the radial symmetry, it is appropriate to impose Neumann boundary conditions at the centre of the bean, namely,

$$
\frac{\partial p_{v}}{\partial r}=\frac{\partial p_{c}}{\partial r}=\frac{\partial T}{\partial r}=0 \text { at } r=0 .
$$

Finally, the initial conditions at $t=0$ are

$$
T=0, p_{v}=p_{v, 0}, p_{c}=p_{c, 0}, S=S_{0} .
$$




\begin{tabular}{ccc}
\hline $\begin{array}{c}\text { Dimensionless } \\
\text { Parameter }\end{array}$ & $\begin{array}{c}\text { Relationship to Dimensional } \\
\text { Parameters }\end{array}$ & Typical value of Parameter \\
\hline$\kappa_{1}$ & $\frac{I_{c 0}}{I_{0}}$ & 1.56 \\
$\kappa_{2}$ & $\frac{\frac{m_{v}}{m_{C}}}{\kappa_{3}}$ & $\mu_{g 0}$ \\
$\kappa_{3}$ & $\frac{k_{w} p_{w 0}}{\mu_{w 0}} \cdot \frac{\mu_{g} p_{g 0} p_{0}}{k_{v}}$ & - \\
$\kappa_{4}$ & $\frac{\lambda_{c}}{\lambda_{v}}$ & - \\
$\mathscr{C}_{3}$ & $\frac{C_{p c}}{C_{p w}}$ & $2.1 \times 10^{-4}$ \\
$\mathscr{D}_{1}$ & $D_{\mathrm{eff}, \mathrm{g} 0} \cdot \frac{\mu_{g 0}}{k_{g 0} p_{0}}$ & - \\
$\mathscr{D}_{2}$ & $D_{w 0} \cdot \frac{\mu_{w 0}}{k_{w 0} p_{0}}$ & - \\
$H$ & $\frac{h_{c}}{h_{v}}$ & - \\
$\zeta_{4}$ & $\frac{K_{C}}{\rho_{w} C_{p w}} \cdot \frac{\rho_{w}}{L^{2} I_{v 0}}$ & $1.0 \times 10^{-8}$ \\
\hline
\end{tabular}

Table 4: Description and typical values of other dimensionless parameters, not used in the simplified form of Model 2. Dimensional parameter values are given in Table 2.

Tables 3 and 4 provide a description and a typical value of each dimensionless parameter shown above. Additionally, we define Model 2 as the PDEs (30), (31), (32), (33), (34), the constitutive equations (35), (36), (37), the boundary conditions (38), (39), (40), and the initial conditions (41).

\subsection{Simplification of Model 2}

While Model 2 presented in Sections 4.3 appears to account for most of the processes relevant to the roasting of coffee beans, there are several difficulties in practice, primarily due to the lack of sufficient relevant physical data for determining realistic values of some parameter values. Furthermore, the complexity of the model makes interpretation of the behaviour of solutions somewhat complicated. In consequence, we are motivated to simplify Model 2 in order to to gain a preliminary understanding of the behaviour of its solutions.

Firstly, we note that several parameter values relating to $\mathrm{CO}_{2}$ gas production are not well documented and that such parameters might only be determined by further experiments. We note however that, at least initially, we anticipate $\mathrm{CO}_{2}$ production to be small and might therefore neglect its influence on the behaviour. This is equivalent to assuming the non-dimensional parameters $\left(\kappa_{1}, \kappa_{2}, \kappa_{4}, \mathscr{C}_{3}, H, \zeta_{4}\right)$ are all small. By doing so, this in turn implies that $\phi$ remains constant.

In addition to the neglecting any $\mathrm{CO}_{2}$ gas transport, we also simplify the transport of liquid water and water vapour. If we assume that the dominant transport of water vapour is pressuredriven rather than by Fick's Law, we can conclude that the parameters $\mathscr{D}_{1}$ and $\mathscr{D}_{2}$ are small and can be neglected. Additionally, since $k_{g 0}$ is very small, due to the low permeability of the nano-porous coffee bean walls, we assume that $\frac{k_{w 0}}{\mu_{w 0}} \ll \frac{k_{g 0}}{\mu_{g 0}}$ (i.e. $\kappa_{3}$ is negligible).

Some parameter values in Model 2 have been determined, but are much smaller than other parameter values in the equation. Since $\alpha_{2} \mathscr{C}_{2} \ll \alpha_{1} \mathscr{C}_{1}$ and $\alpha_{2} \mathscr{C}_{2} \mathscr{D}_{3} \approx 10^{-12}$, we neglect both of these terms. Finally, we assume that $k_{g}^{*}$ and $\mu_{g}^{*}$ are identically constant, implying that $\frac{k_{g}}{\mu_{g}} \equiv 1$, since $k_{g 0}$ and $\mu_{g 0}$ have already been incorporated in the non-dimensionlisation. Combining all of these simplifications, our system of PDEs becomes

$$
\phi \frac{\partial S}{\partial t}=-I_{v}
$$

$$
\begin{gathered}
\phi \frac{\partial}{\partial t}\left(\frac{(1-S) p_{v}}{1+\mathscr{T} T}\right)=\frac{1}{\alpha_{2}} I_{v}+\mathscr{D}_{3} \nabla \cdot\left(\frac{p_{v} \nabla p_{v}}{1+\mathscr{T} T}\right) \\
\alpha_{1} \mathscr{C}_{1}(1-\phi) \mathscr{T} \frac{\partial T}{\partial t}+\phi \frac{\partial}{\partial t}(S(1+\mathscr{T} T)) \\
=-\gamma I_{v}+\mathscr{T}\left(\left(\zeta_{1}(1-\phi)+\zeta_{3} \phi\right) \nabla^{2} T+\left(\zeta_{2}-\zeta_{3}\right) \phi \nabla \cdot(S \nabla T)\right) .
\end{gathered}
$$

In non-dimensional form,

$$
I_{v}=\phi^{2} \frac{\left(p_{S T}-p_{v}\right) S(1-S)}{\sqrt{1+\mathscr{T} T}} \text { and } p_{S T}=B_{1} \exp \left(\frac{B_{2} \mathscr{T} T}{1+\mathscr{T} T}\right)
$$

Additionally for our simplified model, since we are only considering the vapour pressure, our boundary condition for pressure becomes

$$
p_{v}=1+\mathscr{T} \text { at } r=1
$$

and our boundary condition for heat becomes

$$
\frac{\partial T}{\partial r}=\mathrm{Nu}_{v} \zeta_{3}(1-T) \frac{\phi(1-S)}{\zeta_{1}(1-\phi)+\zeta_{2} \phi S+\zeta_{3} \phi(1-S)} \text { at } r=1 \text {. }
$$

Additionally, we preserve the Neumann boundary conditions

$$
\frac{\partial p_{v}}{\partial r}=\frac{\partial T}{\partial r}=0 \text { at } r=0,
$$

as well as the initial conditions at $t=0$,

$$
T=0, p_{v}=p_{v, 0}, S=S_{0}
$$

We define the Simplified Model 2 as the PDEs (42), (43), (44), the constitutive equation (45), the boundary conditions (46), (47), (48), and the initial conditions (49).

\subsection{General Behaviour of Simplified Model 2}

The previous simplification of Model 2 has been done to allow progress to be made and are reasonable for the drying part of the roasting process. However, it is clear that some mechanisms may still dominate others as the remaining parameters in the model still range over several orders of magnitude. A formal asymptotic analysis, exploiting these parameter sizes, remains to be completed but here we indicate the general structure of the behaviour. We note that a typical roasting situation has $\theta \approx 10^{-3} s, \alpha_{2} \approx 10^{-3}, \frac{\mathscr{D}_{3}}{\phi}=\left(\frac{1-\phi}{\phi} \zeta_{1}-\zeta_{3}\right)=\left(\zeta_{2}-\zeta_{3}\right) \approx 10^{-6}$, $\gamma=\mathscr{T}=\frac{1-\phi}{\phi} \alpha_{1} \mathscr{C}_{1} \approx 1$. We note that these parameter values indicate there might be phenomena at three distinct orders of magnitude, namely, $O(1), O\left(10^{-3}\right)$, and $O\left(10^{-6}\right)$. If we introduce a small parameter $\epsilon=O\left(10^{-3}\right)$, then the phenomena are of size $O(1), O(\epsilon)$, and $O\left(\epsilon^{2}\right)$. Making this substitution of $\epsilon$ into our simplified parameter values gives us

$$
\begin{gathered}
\frac{\partial S}{\partial t}=-\phi \frac{\left(p_{S T}-p_{v}\right) S(1-S)}{\sqrt{1+T}} \\
(1+S) \frac{\partial T}{\partial t}=-T \frac{\partial S}{\partial t}+\epsilon^{2} \nabla^{2} T+\epsilon^{2} \nabla \cdot(S \nabla T) \\
\left(\frac{1-S}{1+T}\right) \frac{\partial p_{v}}{\partial t}=\left(\frac{p_{v}}{1+T}-\frac{1}{\epsilon}\right) \frac{\partial S}{\partial t}+\frac{(1-S) p_{v}}{(1+T)^{2}} \frac{\partial T}{\partial t}+\epsilon^{2} \nabla \cdot\left(\frac{p_{v} \nabla p_{v}}{1+T}\right) .
\end{gathered}
$$




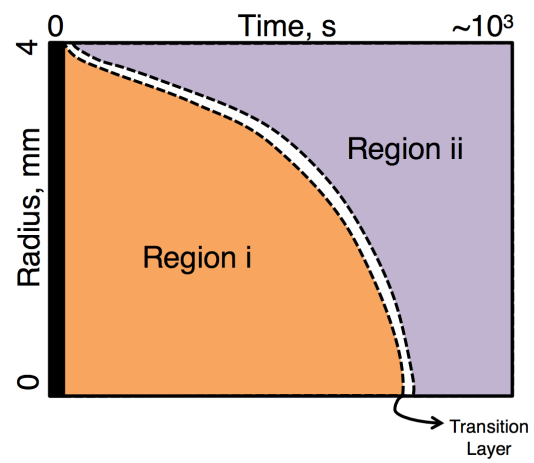

Figure 7: A summary of where the different regions are as the bean dries. Region $\mathrm{i}$ is when the vapour pressure is in equilibrium, Region ii is the dry region, and the dashed lines indicate the narrow transition layer between the regions. There is an initial rapid transient indicated by the black region.

Again, $p_{S T}=B_{1} \exp \left(B_{2} \frac{T}{1+T}\right)$, since $\mathscr{T}=1$ in this further simplified version of the problem.

The main conclusion to be drawn from this formulation of the problem comes from observing that the $-\frac{1}{\epsilon} \frac{\partial S}{\partial t}$ term in (52) appears to be much larger than any of the other terms in that equation. This therefore requires that to lowest order $\frac{\partial S}{\partial t}=0$. In consequence, (50) implies that our modified evaporation rate $\phi \frac{\left(p_{S T}-p_{v}\right) S(1-S)}{\sqrt{1+T}}$ must also be zero to leading order. This requirement can only be satisfied in three ways: i) by having $p=p_{S T}$, ii) by having $S=0$, or iii) by having $S=1$. This naturally divides the solution into various subregions with one region for each of the cases. Physically, we can readily interpret the regions since i) is a region where the water vapour pressure is in equilibrium with the water, ii) is a region that is completely dry and iii) corresponds to a completely water filled part of the bean. Between these regions there will be narrow "transition layers" allowing the behaviour to smoothly vary. In practice, region iii) can only occur if some part the bean is initially full of water (ie $S_{0} \equiv 1$ ) and we do not expect this to occur, hence in subsequent numerical results we will see the development of the two regions, i) and ii). In some situations, the initial or boundary data may not be consistent with the regions i) and ii) described above and in this case there may be some rapid adjustment of the behaviour. A summary of these regions is depicted in Figure 7.

\subsection{Results of Simplified Model 2}

We focus on the Simplified Model 2 for preliminary results. We solve the non-dimensionalised model presented in Section 4.4 using the method of lines with a finite volume scheme in the radial component, as outlined in (12), and a stiff ODE solver for the time stepping (i.e. the MATLAB function ode15s). This was implemented using MATLAB [12] with 75 spatial meshpoints, as a more refined spatial meshing would result in a prohibitive expense of computation time. Lastly, we assume parameters values given in Tables 2 and 3.

In order to compare the experimental data and average moisture concentration presented in [6] with the predictions of Simplified Model 2 we need to briefly describe how the data relates to the moisture content variable in the Simplified Model 2. We can define the moisture concentration $c^{*}$ as the moisture content

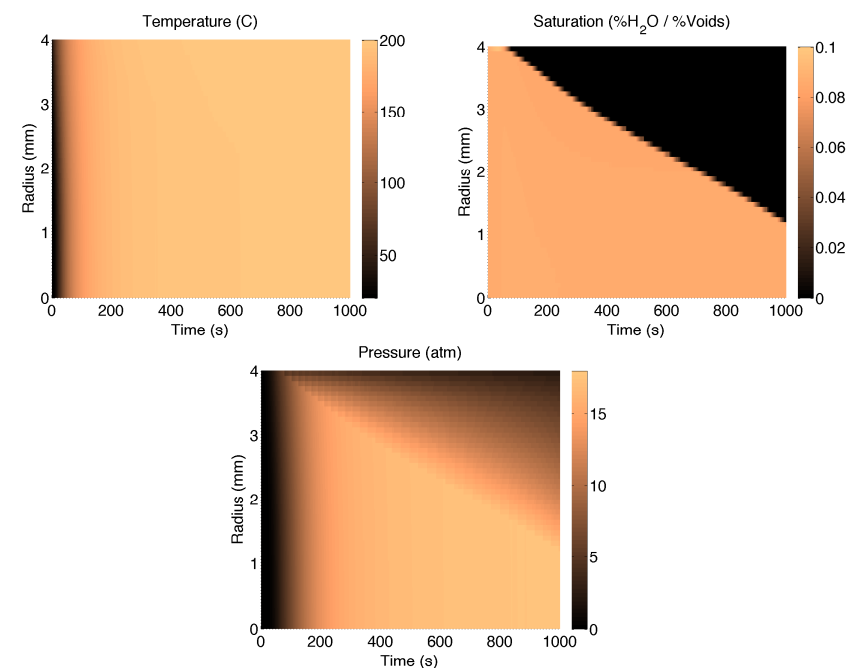

Figure 8: The solutions of Simplified Model 2 for 1000 s of roasting at $200^{\circ} \mathrm{C}$. Dimensionless parameters are specified in Table 3 and boundary conditions described in Section 4.4 are imposed.

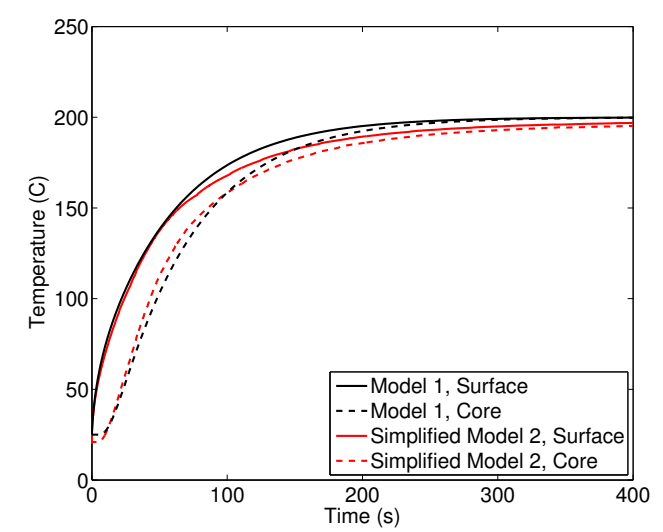

Figure 9: Comparison of temperature curves for a $4 \mathrm{~mm}$ bean at the surface and the centre of the bean during 400 s of roasting at $200^{\circ} \mathrm{C}$ under both Model 1 and the Simplified Model 2. Parameter values for the Simplified Model 2 are listed in Table 3.

$\left(\frac{\phi S \rho_{w}}{(1-\phi) \rho_{s}}\right.$, Section 4.1.5) multiplied by the molar density of water $\left(\frac{\rho_{w}}{m_{w}}\right)$. This gives us

$$
c^{*}=\frac{\phi S \rho_{w}^{2}}{(1-\phi) \rho_{s} m_{w}} .
$$

This in turn tells us that a coffee bean with $12 \%$ moisture initially $\left(5500 \mathrm{~mol} / \mathrm{m}^{3}\right.$, as per [6]) would have $S_{0} \approx 0.084$.

From the numerical results presented in Figure 8, we can see that $S$ forms two distinct regions, as was predicted in Section 4.5. Additionally, in Figure 9, we see that Model 1 and the Simplified Model 2 agree well with the temperature profiles shown at the core and the surface of the bean, when using parameters described in Table 3. Finally, we see in Figure 10 that the average moisture content predicted by the Simplified Model 2 has a very good agreement with the experimental data provided in [6], and affords a better fit than obtained from Model 1.

The most noticeable difference between the $c_{\text {avg }}^{*}$ curves obtained by our two models is that at the beginning of the roast, we observe a slight increase in average moisture concentration in 


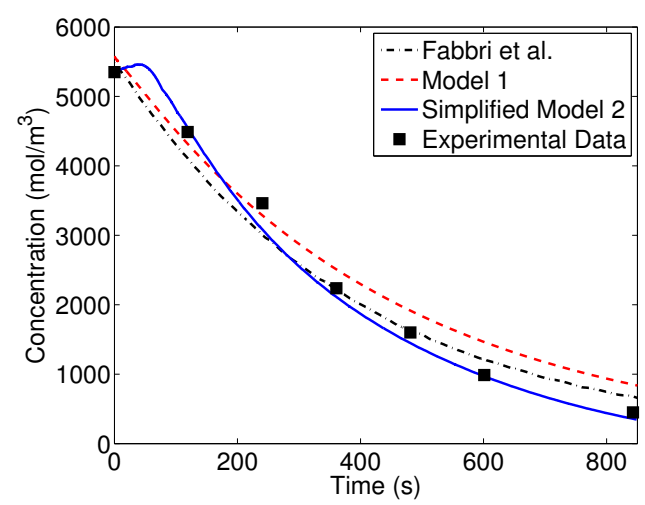

Figure 10: Comparison of average moisture content for a $4 \mathrm{~mm}$ bean during $850 \mathrm{~s}$ of roasting at $200^{\circ} \mathrm{C}$ for simulated and experimental data reproduced from [6], Model 1, and the Simplified Model 2. Parameter values used when obtaining solutions for Model 1 and Simplified Model 2 are listed in Tables 1 and 3 respectively. We also compare our results to those of Fabbri et al. [6].

the solution to the Simplified Model 2, before the bean's moisture starts to evaporate. This is to be expected, since the bean starts out below the boiling point of water at ambient roasting pressure. In consequence, there will be a brief time where water condenses on the bean, thereby raising the moisture content.

\section{Comparison of Solutions to the Two Models}

At this point, it is natural to compare how the existing and new model compare and discuss their relative merits. Indeed, the results shown in Sections 3.3 and 4.6 indicate that both models can be fit to the experimental data shown in [6]. We note that, as indicated in Section 3.3, Model 1 can show numerical ill-posedness if the parameter values are not well-chosen. This behaviour does not seem to occur with the Simplified Model 2. Regarding the internal transport of water, Model 1 uses a simple mass diffusivity term, while the Simplified Model 2 considers vapour and liquid water as separate entities. In the former case, there is a need to ensure that the diffusivity is correctly fitted to data in the relevant temperature range since it originates from models below the boiling point [8], while the latter suffers from a lack of physical data to properly quantify the many more parameters that are required. In particular, the Simplified Model 2 currently uses an ideal "steam table" pressure for the vapour pressure and this needs significant modification in the case of water bound to the solid phase. One shortcoming of the specific mass diffusivity used in the Model 1 is its dependence on the average moisture content. Such a dependency gives good fitting of the data, but the underlying physical mechanisms that it represents are not clear.

Although there is agreement on the predicted average moisture in a bean, there are substantial differences between the two models concerning the predicted distribution of the moisture the coffee bean. Indeed, while both models predict a drying front that moves to the centre of the bean, the moisture content remaining after the drying front varies significantly. In Model 1 , the moisture in the bean slowly decreases after the drying front has reached the centre of the coffee bean, and the moisture

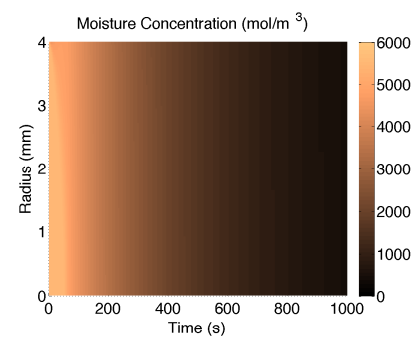

(a)

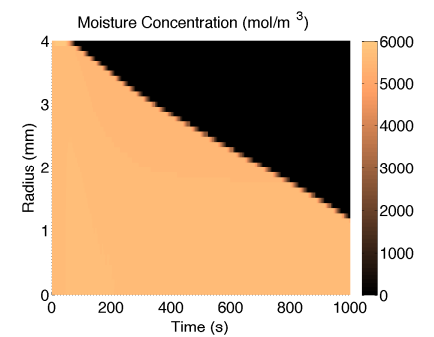

(b)
Figure 11: Comparison of moisture concentrations in a $4 \mathrm{~mm}$ bean during $1000 \mathrm{~s}$ of roasting at $200^{\circ} \mathrm{C}$ for (a) Model 1 and (b) the Simplified Model 2. Parameter values for Model 1 and Simplified Model 2 are listed in Tables 1 and 3 respectively.

throughout the bean is nearly uniform. On the other hand, in the Simplified Model 2, the coffee bean is distinctly divided into a "moist" region (where $S \sim S_{0}$ ) and a dry region $(S \sim 0$ ). Additionally, the drying front in the Simplified Model 2 is much slower than in Model 1. This is because Model 1 measures moisture in the bulk, and will show a bean drying in average moisture content, while Model 2 will show the progress of the drying of a bean locally. Due to evaporation being a local phenomenon, we conclude that Model 2 has a more realistic drying front. Such a comparison of these two models is shown in Figure 11. Note that these differences may play a significant role if used to predict where chemical reactions in the coffee bean can occur. In consequence, having a different localized model for the moisture concentration inside a coffee bean can significantly vary the predictions of other chemical reactions that can occur in a bean.

Until now, our main conclusion of the two models is that, while predicting different local moisture content within the bean, one can present similar average moisture content values to what is seen experimentally. We will now investigate how sensitive these parameters are to the lengthscale chosen using the following thought experiment. Suppose that we choose to coarsely chop up our green coffee beans and then roast them, rather than the standard method of grinding beans after roasting. Without changing any of the other parameters in the two models, how do the results differ? To answer this, we solve Model 1 and Simplified Model 2 as before, but with changing $L=0.2 \mathrm{~mm}$ to reflect a coarse chopping of the bean. As we can see in Figure 12, the temperature profiles produced from Model 1 and Simplified Model 2 are nearly identical. However, we also note that the moisture content predicted by these two models differ considerably. Indeed, Model 1 predicts a bean chunk that is nearly at roasting temperature, but still has a high moisture content. Conversely, Simplified Model 2 predicts that after approximately $3 \mathrm{~s}$, the entire bean chunk has zero moisture content. While experimental data on similar operating conditions have not been found, we can still conclude from this thought experiment that Model 1 and Simplified Model 2 will not always produce similar results in terms of average moisture content. 


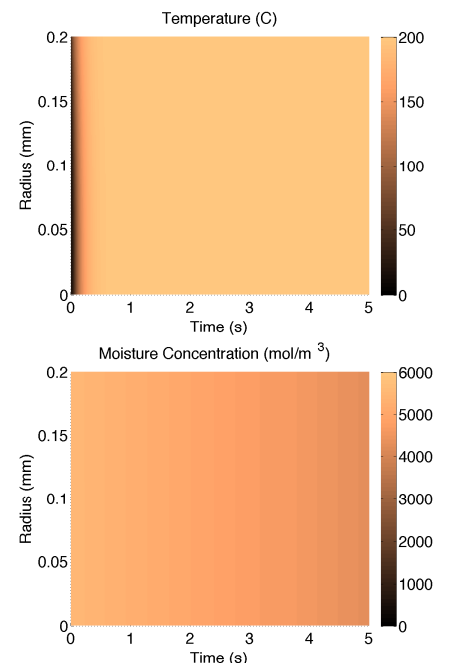

(a)

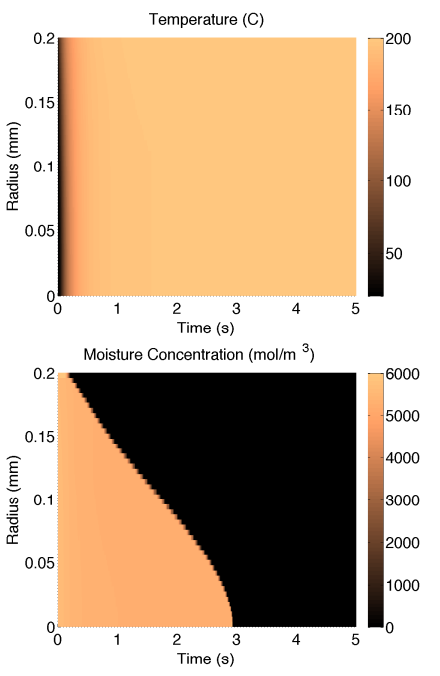

(b)
Figure 12: Comparison of moisture concentrations in a $0.2 \mathrm{~mm}$ bean chunk during $5 \mathrm{~s}$ of roasting at $200^{\circ} \mathrm{C}$ for (a) Model 1 and (b) the Simplified Model 2. Parameter values for Model 1 and Simplified Model 2 are listed in Tables 1 and 3 respectively.

\section{Conclusions}

We studied an existing model from the literature of the roasting of coffee beans and analysed it by looking at a very similar model, namely, Model 1 . With a suitable nondimensionalisation, we were able to fit parameters occurring in this model to match the experimental data. While the parameters in the model could be adjusted to get a reasonable fit on the data, the model showed potential numerical problems when parameters were chosen slightly differently. A new model (Model 2) was derived from conservation equations accounting for movement of liquid water and various gases. By nondimensionalising this system, as well as making certain simplifications to the model, we were also able to fit the experimental data obtained in [6] using the simplified form of Model 2. The main drawback of this model is the lack of physical data to properly fit the numerous parameters, since not all parameters have been previously determined experimentally, and this is ongoing work. There were substantial differences between the models in the predicted distribution of moisture during the drying of the bean, and this needs to be investigated further. Compared to existing studies in the literature, out first principles model (Model 2 and its simplification) allows for the influence of local moisture and heat transfer on the coffee bean roasting process to be better understood. Importantly, including these local influences in our model, we were able to see the emergence of an explicit drying front, which was not previously seen in the literature when bulk quantities were employed. Therefore, our first principles model can prove useful to various roasting applications within the coffee industry, and also for related roasting problems for which the roasting mechanism depends strongly on the existence of a drying front which penetrates from the surface to the core of a material.

Now that the first principles model is derived, there are two clear directions to be considered next. The first would be a sys- tematic simplification of the model and exploiting asymptotic methods to quickly determine trends in behaviour. This work is in progress, and such an asymptotic study in particular will case more light on the qualitative dynamics of the drying front, as well as the processes occurring in front of and behind the drying front. The second natural extension would be an exploration of the effects of other aspects of the roasting process, such as the role of $\mathrm{CO}_{2}$ or other chemical reactions, particularly in the later stages of roasting. Including even further chemistry would be useful when incorporating solid mechanics effects, which need to be considered when the bean develops fractures and cracks after heating.

\section{Acknowledgments}

This publication is based on work supported by the EPSRC Centre for Doctoral Training in Industrially Focused Mathematical Modelling (EP/L015803/1) in collaboration with Jacobs Douwe Egberts. N. Fadai thanks Jacobs Douwe Egberts for financial support and the opportunity to work on-site during parts of this project. The authors wish to thank I. Hewitt for helpful discussions.

\section{References}

[1] Baggenstoss, J., 2008. Coffee roasting and quenching technology - Formation and stability of aroma compounds. PhD thesis, Swiss Federal Institute of Technology.

[2] Bird, R.B., Stewart, W.E., and Lightfoot, E.N., 1960. Transport Phenomena. John Wiley and Sons, Inc., New York.

[3] Chandrasekar, V., and Viswanathan, R. 1999. Physical and thermal properties of coffee. Journal of Agricultural Engineering Research 73, 227234.

[4] Comsol, A.B. 2005. COMSOL multiphysics users guide. Version: September.

[5] Dean, J.A., Ed. 1999. Lange's Handbook of Chemistry, 15th Ed., McGraw-Hill, New York.

[6] Fabbri, A., Cevoli, C., Alessandrini, L., and Romani, S. 2011. Numerical modeling of heat and mass transfer during coffee roasting process. Journal of Food Engineering 105, 264-269.

[7] Fick, A. 1855. On liquid diffusion. Poggendorffs Annalen 94, 59.

[8] Hernandez-Diaz, W.N., Ruiz-Lopez, I.I., Salgado-Cervantes, M.A., Rodriguez-Jimenez, G.C., and Garcia-Alvarado, M.A. 2008. Modeling heat and mass transfer during drying of green coffee beans using prolate spheroidal geometry. Journal of Food Engineering 86, 1-9.

[9] Hsu, C.T., and Cheng, P. 1990. Thermal dispersion in a porous medium. International Journal of Heat Mass Transfer 33, 1587-1597.

[10] Langmuir, I. 1919. The Vapour Pressure of Metallic Tungsten. Physical Review 2, 329-342.

[11] Larini, M., Giroud, F., Porterie, B. and Loraud, J.C., 1998. A multiphase formulation for fire propagation in heterogeneous combustible media. International Journal of Heat and Mass Transfer 41, 881-897.

[12] MATLAB, 1990. Users' Guide and Statistics Toolbox Release. "Natick." MA: The Math Works.

[13] Moroney, K.M., Lee, W.T., O’Brien, S.B.G., Suijver, F., Marra, J. 2015. Modelling of coffee extraction during brewing using multiscale methods: an experimentally validated model. Chemical Engineering Science 137, 216-234.

[14] Ni, H., Datta, A.K., and Torrance, K.E. 1999. Moisture transport in intensive microwave heating of biomaterials: A multiphase porous media model. International Journal of Heat and Mass Transfer 42, 1501-1512.

[15] Paik, S., and Nguyen, H. D. 1995. Numerical modeling of multiphase plasma/soil flow and heat transfer in an electric arc furnace. International Journal of Heat and Mass Transfer 38, 1161-1171.

[16] Pečenko, R., Svensson, S., Hozjan, T. 2015. Modelling heat and moisture transfer in timber exposed to fire. International Journal of Heat and Mass Transfer 87, 598-605.

[17] Ruiz-Lopez, I.I., Cordova, A.V., Rodriguez-Jimenez, G.C., and GarciaAlvarado, M.A. 2004 Moisture and temperature evolution during food 
drying: effect of variable properties. Journal of Food Engineering 63, 117124.

[18] Schenker, S. 2000. Investigations on the hot air roasting of coffee. $\mathrm{PhD}$ thesis, Swiss Federal Institute of Technology, Zurich.

[19] Séro - Guillaume, O., \& Margerit, J. 2002. Modelling forest fires. Part I: a complete set of equations derived by extended irreversible thermodynamics. International Journal of Heat and Mass Transfer 45 1705-1722.

[20] Talbot, J.M. 2004. Grounds for agreement: The political economy of the coffee commodity chain. Rowman and Littlefield, Oxford, UK.

[21] Wang, X., and Lim, L.T. 2014. A kinetics and modeling study of coffee roasting under isothermal conditions. Food and Bioprocess Technology 7, 621-632.

[22] Wang, C. Y., and Cheng, P. 1996. A multiphase mixture model for multiphase, multicomponent transport in capillary porous media - I. Model development. International Journal of Heat and Mass Transfer 39, 36073618.

[23] Zhang, J., and Datta, A.K. 2006. Mathematical modeling of bread baking process. Journal of Food Engineering 75, 78-89. 